\title{
Looping with Do-It-Yourself Artificial Pancreas Systems During Ramadan Fasting in Type 1 Diabetes Mellitus: Perspectives of a User and a Physician
}

Syed Haris Ahmed (D) - Saira Gallo

Received: July 21, 2020 / Published online: September 9, 2020

(C) The Author(s) 2020

\section{ABSTRACT}

Hybrid closed-loop automated insulin delivery systems have helped type 1 diabetes (T1D) users close the loop between glucose monitoring and insulin delivery, a very important step in efforts to simulate the glucose-responsive insulin secretory function of a healthy pancreas. Do-ItYourself Artificial Pancreas Systems (DIY APS) are a form of hybrid closed-loop system that use open-source algorithms, which govern the delivery of insulin in response to interstitial

Digital Features To view digital features for this article go to https://doi.org/10.6084/m9.figshare.12727640.

S. H. Ahmed (两)

Department of Endocrinology and Metabolic Medicine, Countess of Chester Hospital NHS

Foundation Trust, Chester, UK

e-mail: asyedharis76@gmail.com

S. H. Ahmed

School of Medicine, University of Liverpool, Liverpool, UK

S. Gallo

Tustin, California, USA glucose and other variables that are personalized to an individual. The flexibility and customization afforded by these systems make them amenable for use in different worldly circumstances, one of which is fasting during the annual occurrence of Ramadan for observant Muslims. Here, we present the views of a DIY APS user who was able to fast successfully on most days of Ramadan after adopting this system, and the overview of a physician on these systems, with a focus on fasting during Ramadan with T1D.

Keywords: Artificial pancreas systems; Do-ityourself; Hybrid closed-loop systems; Iftaar; Looping; Ramadan; Suhoor; Time-in-range; Type 1 diabetes; \#WeAreNotWaiting 


\section{Key Summary Points}

Observing Ramadan fasting is an important pillar of faith and hence holds deep spiritual value for practicing Muslims. However Type 1 diabetes patients are risk stratified as being at high risk, because of the potential for acute complications and hence advised not to fast.

In this commentary, a Type 1 diabetes patient shares her experience of using a Do-It-Yourself Artificial Pancreas System (DIY APS), to manage her diabetes during Ramadan fasting. Her quality of life and ability to self-manage diabetes were greatly enhanced by this open source technology.

DIY APS, a brainchild of the \#WeAreNotWaiting community, has the flexibility and customizability that are required for a fasting Type 1 patient; hence these systems are a therapeutic option for patients who choose to fast for religious or personal reasons.

Further research would help regulatory bodies develop a framework and guidance, for health care professionals to support DIY APS users.

\section{A T1D USER PERSPECTIVE}

I was diagnosed with type 1 diabetes in early 1996, when I was 8 years old and living in Pakistan. When I woke up from my diabetic ketoacidosis (DKA) coma, one of the first things I was told was a list of the things I would never be allowed to eat again. All the kids at my school were told I was contagious, so overnight I lost any friends I had made with great difficulty as a shy child.

Because of this, management of my type 1 diabetes lay on the periphery of my life for many years. During my high school years while living in Kuwait, I suffered from diabulimia. I did the bare minimum to survive, instead of to thrive. I moved back to the United States for university, before joining the US Peace Corps. This is when I happened to meet my now husband through our matching insulin pumps and finally had the community and motivation to take better care of myself. A few years later, our first daughter, Aiyla, was also diagnosed with type 1 diabetes when she was 10 months old.

With three T1D patients in our household, I desperately needed a way to manage all our blood sugars while also keeping my sanity. We got my husband the Medtronic MiniMed 670G system (insurance was not ready for me to upgrade yet). And then I came across the idea of the DIY Loop. I decided to try looping because I needed to focus all my energy on managing our daughter's type 1 diabetes and I had noticed that my good days always correlated with her bad ones and vice versa.

After some research, I decided on Loop instead of OpenAPS or AndroidAPS, mainly because I was already an iPhone user and happened to have a compatible legacy Medtronic pump on hand. All I needed was to purchase a RileyLink, create an Apple Developer account and download Xcode. It felt like a pretty minimal amount of effort for the potential impact this software could have on my life. Looping made it so that my type 1 diabetes took up a lot less headspace and brainpower for me, while bringing my HbA1c down to 5.6\%. It also gave me the ability to take care of Aiyla the way I needed to. It also allowed me to begin practicing a pillar of my faith in a way I had never been able to before.

All over the world, Muslims celebrate the month of Ramadan by fasting from dawn until sunset. A meal is eaten pre-dawn called 'Suhoor;' towards the end of a typical fasting day, when the sun goes down, they break their fast over the 'Iftaar' meal. It is a big milestone when a child fasts for the first time, and an even bigger one when they keep the fasts for the entire month. Most children will try to keep their first fast between the ages of 7-10. It is not required until after puberty. It is also not required of women who are pregnant or nursing, the elderly and sick. If you are physically 
unable to because of an ailment, you are exempt from this pillar and are asked to instead feed a person for every day that you cannot fast or make a donation to feed the needy.

Ramadan is a very magical time for practicing Muslims, because fasting and keeping away from our physical needs is a form of spiritual cleansing. It allows us to focus on prayer, on charity, on being kind and on the fact that we are blessed enough to know that at sunset we will have food and water, whereas so many poverty-stricken people do not have that luxury. Ramadan is a month of personal reflection and betterment, a time of community and celebration. It is a time to remember who and what are truly important in life.

Because of how old I was at diagnosis, I never got to experience this big milestone. As a diabetic, and with the long days, it can wreak havoc on our blood sugars to try to fast all day. Both my husband and I had tried, but we would end up having to take no insulin for the second half of the day, only to shock our systems after the breaking of the fast, which skyrocketed our blood sugars, keeping them $\geq 16 \mathrm{mmol} / \mathrm{l}$ the rest of the night. We're pretty sure God does not want us to hurt our bodies, so we stopped trying.

Then, last year, after I started looping, I tried again. And this time, I succeeded far more days than I did not. I had been looping for a while and felt like I had my settings pretty dialed in. I figured it would be like a really good basal test. I was wrong about that, because your settings change after changing your routine so drastically for so long. But I safely kept a little over 20 out of 30 days last year, the rest of them only being broken by a few skittles or smarties. This year, I kept closer to 25 out of 30 days thanks to a deeper understanding of how the Loop works, and the updates to the system, specifically around the overrides feature.

Just like last year, it took a while for my body to adjust to the new routine as far as eating so early in the morning and eating a big meal later than normal. Eating that early and that late in the day was an adjustment for my body, and figuring out my insulin carbohydrate ratios (ICRs) for those times took some trial and error.
In the first week of Ramadan, I used a "Ramadan" override in Loop with a 30\% decrease in all my settings during the day and another "Post-Iftaar" override with a $140 \%$ increase to prevent the post-Iftaar spike along with a good 20-min pre-bolus. I struggled with going low at night even though Loop had been cutting off my insulin for a few hours and I wasn't entering in my correction carbohydrates (Fig. 1). I thought the post-Iftaar override was too aggressive and catching up with me later at night, even though it seemed to work fine until 10 p.m. I was also running on the high side in the morning after Suhoor. I changed a few settings-lowered my insulin sensitivity factor (ISF) value in those post-Suhoor hours and also increased my basal.

The second week, I cut out the post-Iftaar override. I'm pretty sure I was being too aggressive with the override, which is what was causing my nighttime lows. Instead, I changed ICR for the evening by a bit (made that a little more aggressive) and tried to be really good about using a pre-bolus for at least $30 \mathrm{~min}$ before Iftaar. The highest I went to was $9.5 \mathrm{mmol} / \mathrm{l}$ after Iftaar on the days I wasn't as good about pre-bolusing (kids' bedtimes, preparing Iftaar, etc.). I would trend in the 6.6-7.2 mmol/1 (sometimes going up to 7.7, which is what I have my high alert set to) for the first half of the day; then later in the afternoon, I tend to start to dip a bit but not enough to make me need to break my fast (Fig. 2).

The third week, I started going low at night again. So, I dialed back ICR and ISF, so it wasn't as aggressive at Iftaar anymore. I started using the override right after Suhoor; otherwise, I would go low later (Fig. 3). Pre-bolusing for Suhoor was difficult as this meant that I would have to wake up early, which would affect the duration of my sleep; however, I was usually trending low at that time in the morning, so it seemed to work out. I found that the further we got into Ramadan, the less aggressive I had to be during mealtimes as my body adjusted to the new routine.

This last week of Ramadan has been beautiful. I started turning on the automatic bolus (auto bolus) feature in Loop for post-Iftaar along with a good pre-bolus before eating. I also 


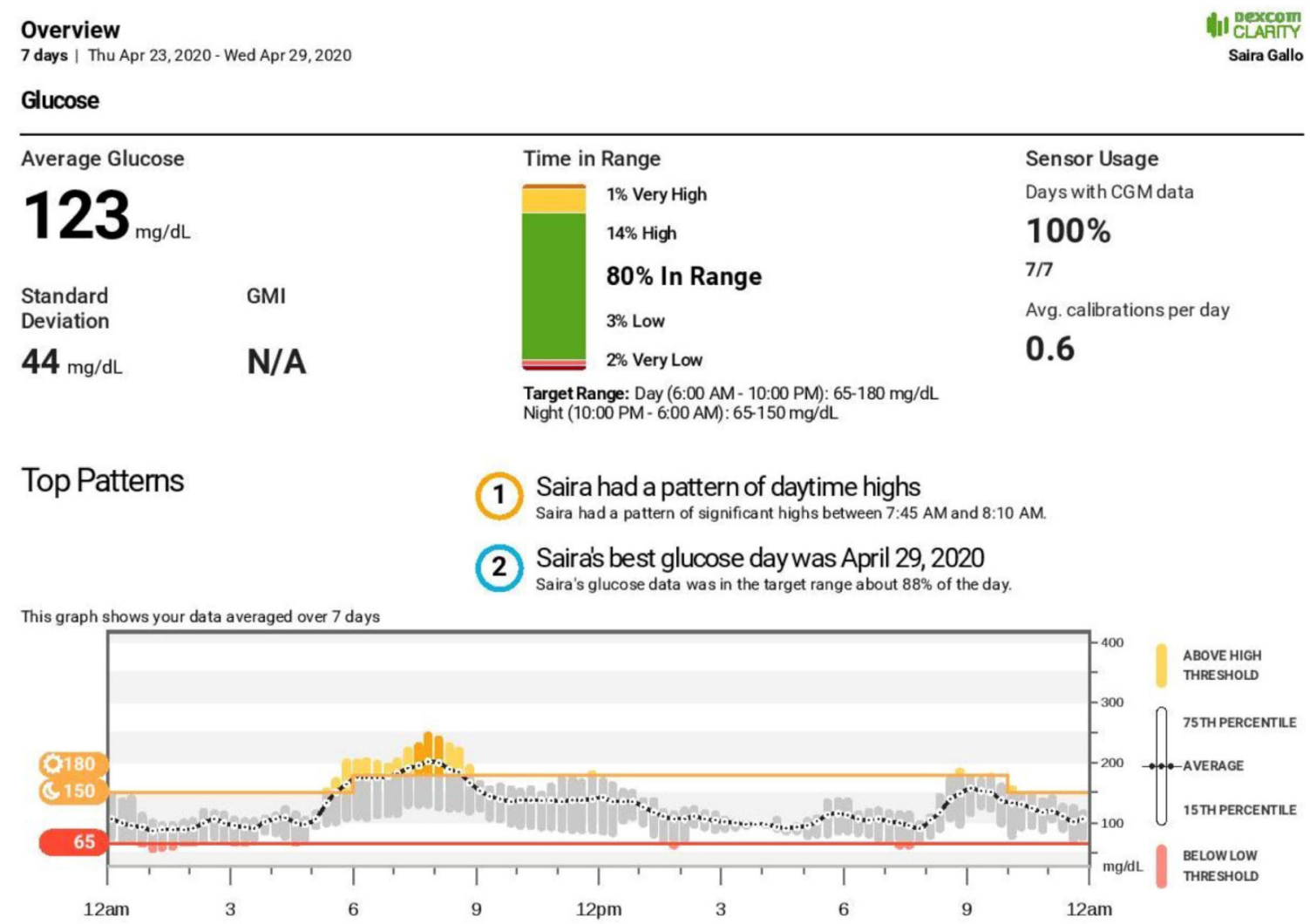

Fig. 1 Overview of Saira's blood sugar readings in the first week of Ramadan: nocturnal dips, due to aggressive post-Iftaar overrides, and elevations post-Suhoor

turned it on during Suhoor and managed not to bolus manually for Suhoor despite eating about $50 \mathrm{~g}$ of carbohydrates in the morning (Fig. 4). But then I turn off the auto bolus feature during the rest of the day and went back to my $70 \%$ override. I didn't need to eat for any of the times I went down to $3.9 \mathrm{mmol} / \mathrm{l}$ because Loop just brought me back into range perfectly. Many Muslims have a medium-large meal for Iftaar, but I learned to break my fast with half a date and water and say my prayers (which gives my insulin a chance to kick in) before skipping right over to dinner. This helps with the postIftaar spike that a lot of diabetics can experience.

Learning from my experience from the previous Ramadan, I used a combination of overrides and changing settings this year unlike last year where I only used overrides. I changed my ISF and ICRs during the new mealtimes because of the need to be more aggressive, but thankfully it is easy enough to go back and adjust those as needed. Basal rates in keeping with my physiology and requirements range from 0.9 to 1.25 units/hour. For Ramadan, I have two different overrides, one that is more cautious (70\%) and the other is $90 \%$. I have my high alert set to $7.7 \mathrm{mmol} / \mathrm{l}$, and when I see it climbing above that I either switch to the $80 \%$ override or just turn it off altogether for a bit. And then if I see I'm trending lower again I just turn it back on, and it has worked for the most part.

The advantages to using the DIY Loop while fasting during Ramadan over the current and widely used closed-loop Medtronic 670G system include:

- Being able to use an extremely reliable and accurate sensor, which is imperative while fasting. Personal experience with Medtronic sensors is that at least $20 \%$ of the time the sensor was off by up to $8.5 \mathrm{mmol}$. With the 


\section{Overview}

7 days | Thu Apr 30, 2020 - Wed May 6, 2020

Glucose

\section{Average Glucose}

$115_{\text {magt }}$

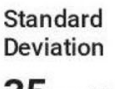

$35 \mathrm{mg} / \mathrm{dL}$

Top Patterns
GMI

N/A

\section{Time in Range}

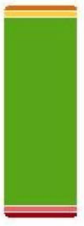

$1 \%$ Very High

$6 \%$ High

91\% In Range

$1 \%$ Low

$<1 \%$ Very Low
Sensor Usage

Days with CGM data

$100 \%$

$7 / 7$

Avg. calibrations per day

0.3

Target Range: Day (6:00 AM - 10:00 PM): $65-180 \mathrm{mg} / \mathrm{dL}$ Night (10:00 PM - 6:00 AM): 65-150 mg/dL

(1) Saira's best glucose day was May 5,2020

Saira's glucose data was in the target range about $100 \%$ of the day.



Fig. 2 Overview of Saira's blood sugar readings in the second week of Ramadan: reduction in nocturnal dips by not using post-Iftaar override but pre-bolusing 30-min before Iftaar and attenuation of elevations post-Suhoor

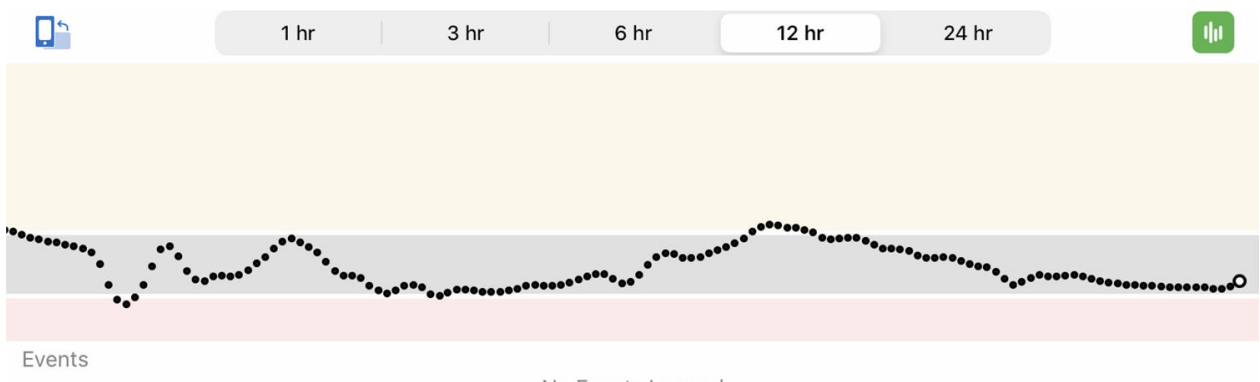

No Events Logged

Insulin

No Insulin Events Logged

\begin{tabular}{lccc}
\hline 3AM & 6AM & 9AM & 12PM Now
\end{tabular}

Fig. 3 A 12-h snapshot of Saira's blood sugar readings between 0200 and 1400 in the third week of Ramadan: physiologic adaptations cause Saira to drop low again at night, for which Saira reduced ICR and ISF to give less insulin for Iftaar; for the post-Suhoor elevations, she used a gentle override immediately post-Suhoor 


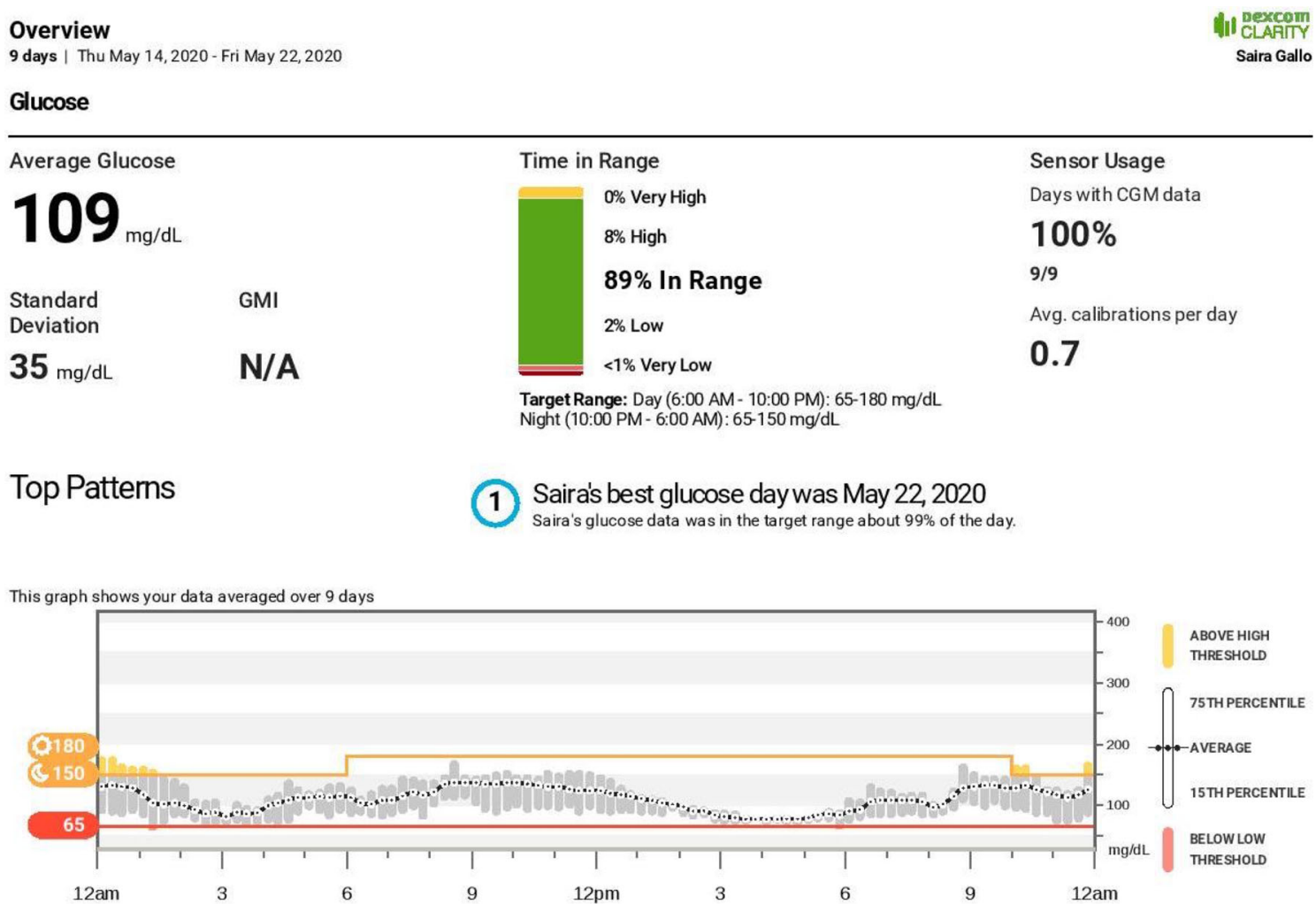

Fig. 4 Overview of Saira's blood sugar readings in the fourth week of Ramadan: further physiologic adaptations occur, with Saira now using a combination of pre-bolus and auto bolus for Iftaar and simply auto bolus for Suhoor

Loop you can choose between any of the Dexcom sensors, which is pretty accurate, and I have been using the G6 since it came out in 2018.

- The ease with which I could customize my settings both during the day while fasting and in the evening when I required significantly different settings.

- The flexibility to change those settings and the override recommendations as needed through the course of Ramadan, as detailed above. The Medtronic 670G offered limited overrides like its exercise mode, which essentially makes it more conservative. However, it does not last long, and you do not really get to set it according to what works for you as easily as you can change settings with Loop with emerging trends. Medtronic has a slower learning curve, which in a shortdefined period of 29/30 days is not ideal; each week can be different as the body adapts itself to a different routine. There is a lack of customizability, which makes it impossible to dial in settings, with different settings each week of the month.

I am grateful to the developers of DIY APS, and I am amazed at the rate at which these systems are evolving, thanks to the enthusiasm of the members of \#WeAreNotWaiting community. The system has allowed me and other T1D Muslim patients I know to fast during the month of Ramadan. The benefits are many, and I hope that, in the not-so-distant future, this technology will be regulated and approved for medical use so that health care specialists can recommend this as a treatment option for $\mathrm{T} 1$ patients.

\section{PHYSICIAN PERSPECTIVE}

Fasting in the Islamic month of Ramadan is obligatory for all sane, healthy adult Muslims. The length of the day varies significantly in 
temperate regions-typically lasting $\geq 18 \mathrm{~h}$ during peak summer in the UK. The synodic nature of the Islamic calendar means that Ramadan migrates through all four seasons over a 33-year cycle. Despite valid exemptions, there is an intense desire to fast during this month even among those who are considered high risk including many individuals with diabetes mellitus.

Diabetes patients wishing to observe the fast are advised to attend a pre-Ramadan assessment with their health care providers (HCPs) at least 6-12 weeks before the start of Ramadan [1]. The International Diabetes Federation and the Diabetes and Ramadan International Alliance (IDFDAR) Practical Guidelines adopt a three-tiered risk stratification matrix, with patients stratified to the very high- or high-risk groups being advised not to fast and remain a well-accepted stratification that can be applied for people with diabetes prior to Ramadan fasting (Table 1) [2]. The guidance highlights the general consensus among religious authorities and scholars that if an individual is considered at high to very high risk then they should refrain from fasting [3]. Factors to be taken into consideration during the assessment for the purpose of risk stratification are type of diabetes, ongoing diabetes treatment, degree of diabetes control, individual propensity for hypoglycemia, competence at self-management, previous Ramadan experience, presence of diabetes complications and comorbidities, ongoing or recent intercurrent illness, degree of frailty, level of cognition, polypharmacy burden, occupation and social circumstances [1]. Diabetes patients can be risk stratified from low, medium to high or very high risk during the preRamadan assessment and counseled accordingly. Those who are high to very high risk are advised not to fast. The current COVID-19 pandemic upgrades those in the high-risk category to very high risk; hence, a significant number of diabetes patients may fall under the penumbra of the 'not-to-fast' advisory [4].

Well-controlled and poorly controlled T1 diabetes patients come under the high-risk and the very high-risk category, respectively, and are advised not to fast. However, if the patient makes an informed decision to fast, an individualized management plan should be developed and they should be supported in the management of their diabetes [1]. The acute challenges that fasting $\mathrm{T} 1$ patients (and T2 patients on insulin) face are the hyperglycemia associated with the two meals, i.e., Suhoor and Iftaar, the hypoglycemia during the fast and the potential for hypoglycemia due to aggressive bolusing for meals or from overcorrection of post-meal spikes.

In $\mathrm{T} 1$ diabetes patients, while fasting has been shown to be feasible in patients on subcutaneous (SC) insulin therapy [5], continuous subcutaneous insulin infusion (CSII) via insulin pumps offers the advantage of allowing a measured delivery of insulin in basal or bolus forms, which can be tailored to different situations of the user. e.g., fasting, work, exercise, etc.

A few of the earliest reported experiences of T1 diabetes patients fasting on CSII therapy have shown that fasting is feasible with adequate counseling, education and support $[5,6]$, without significant adverse events. A multicenter study comparing CSII with multiple-dose insulin (MDI) showed no difference in the rates of severe hypoglycemia, glycemic control or the number of days patients had to be stopped fasting for extreme dysglycemia [7]. There was no ketoacidosis reported, and glycemic variability was better in the CSII group. A systematic review comparing CSII with multiple daily injections (MDI)/premixed insulin regimens has shown lower rates of severe hypoglycemia and hyperglycemia/ketosis with CSII [8].

The use of continuous glucose monitoring (CGM) [9-11] or flash glucose monitoring (FGM) $[11,12]$ along with pre-Ramadan structured education can enhance the advantages offered by CSII in diabetes management during Ramadan fasting, helping observants fast successfully and safely.

Using the low-glucose suspend function in CSII systems can further improve the safety of fasting in adolescents and young adults [13]. Hybrid-closed loop systems offer further potential in improving safety and improved glycemic outcomes by linking CGM and CSII by an algorithm that automates insulin delivery $[14,15]$.

Do-it-yourself (DIY) closed-loop systems or DIY artificial pancreas systems (APS) are 
Table 1 Adapted International Diabetes Federation and the Diabetes and Ramadan International Alliance (IDF-DAR) risk categories and recommendations for patients with diabetes who fast during Ramadan








Fig. 5 The encased RileyLink next to a US quarter for size reference. The RileyLink bridges Bluetooth Low Energy from the phone to the radiofrequency of the pump and vice versa

unregulated hybrid closed-loop systems that use open-source software to allow a piece of hardware called the "rig," which is generally a computing microprocessor, to communicate information via a radio stick, such as Saira's RileyLink (Fig. 5), to and from a pump and also communicate with the CGM directly or via a cloud. The microprocessor collates all the data, which runs through an algorithm, and decides how much insulin to give based on the settings on the pump. Saira's iPhone with the associated Loop app is the "rig" in the Loop system.

Evidence for hybrid closed-loop systems using open-source code is limited to observational studies, self-reported patient outcomes and anecdotal reports with a conspicuous lack of robust clinical trials. Observational data emphasize the usefulness of this system in reducing hypoglycemia, glycemic variability, overnight controls and management burden with improvement in time in range (TIR), HbA1c [16, 17] and sleep [16, 18]. A Twitter analysis of posts from users and carers demonstrated improvement in HbA1c, glucose variability, diabetes burden and quality of life [18]. It has been estimated that users can save up to 1 day's time per month spent making decisions about diabetes management [19].

There is no current framework that governs the accountability and responsibility on health care provision to DIY users. The technology has not been appraised or approved by regulatory bodies such as the National Institute of Clinical Excellence (NICE), Food and Drug Administration (FDA) and Medicine and Healthcare Products Regulatory Agency (MHRA). While current DIY APS users have extensive expertise in diabetes self-care, as the numbers increase, HCP may be asked to support individuals who lack a deeper understanding of the technology [20].

Ramadan is an important annual occurrence for a practicing Muslim, which has a deep spiritual meaning. Significant dietary and lifestyle adjustments are required within a short span of time to observe a fast. The physiologic adaptations that occur as a result can be rigorous, especially in countries with prolonged fasting times or extremes of ambient temperatures. In chronic disease such as diabetes, there is the risk of maladaptive pathophysiology, which can put the fasting individual at high risk of morbidity [1]. It is therefore essential for healthcare professionals and patients to be aware of such risks and take the necessary precautions to minimize them. Hence, we advise a pre-Ramadan assessment $6-12$ weeks before Ramadan [1]. The flexibility, customization and the spontaneity of the Loop allowed Saira to use a variety of features such as overrides, pre-bolus and auto-bolus to combat the challenges of hyperglycemia and hypoglycemia described above. Hopefully, she will use the learning from this year (2020) to fast with a better average TIR in future Ramadans for as many days as she wishes. 
The Medtronic MiniMed $780 \mathrm{G}$ system, an automated insulin delivery system, which secured the CE marking in June 2020 (awaits a commercial launch), has added features, among which the ability to adjust the target setting to as low as $5.5 \mathrm{mmol} / \mathrm{l}$ and Bluetooth connectivity with compatible iOS and Android systems, are prominent [21]. This and other sophisticated systems on the horizon for which clinical trials are underway may parallel the flexibility and customizability of DIY APS, and thus have the potential to serve as medically approved and regulated alternatives for those who wish to fast.

T1 diabetes patients may want to fast for personal, health or religious reasons. Technology has facilitated T1 diabetes patients' ability to self-manage their diabetes while minimizing the risk of harm; the automation of glucose sensing and insulin delivery by current systems provides an opportunity to explore and formally research the prospect of fasting in T1 diabetes. Besides Saira, there are other unpublished accounts of DIY APS users who have managed to fast safely and satisfactorily for religious reasons (such as Ramadan) and for personal or health reasons. Although as healthcare professionals we are not legally obliged or insured to recommend these systems, we should support patients using this technology during fasting (and at other times) to make their fasting experience safe and positively memorable. The use of DIY APS hybrid closedloop systems continues to grow in popularity with patients and their families. It is vital that HCPs have some understanding of these systems and are able to discuss possible risks and benefits with their patients and support them where this is feasible.

\section{ACKNOWLEDGEMENTS}

I thank the participant Ms Saira Gallo for her contribution to this article.

Funding. No funding or sponsorship was received for the write up or publication of this article.
Authorship. All named authors meet the International Committee of Medical Journal Editors (ICMJE) criteria for authorship for this article, take responsibility for the integrity of the work as a whole, and have given their approval for this version to be published.

Disclosures. Syed Haris Ahmed and Ms Saira Gallo have nothing to disclose.

Compliance with Ethics Guidelines. This article is based on previously conducted studies and does not contain any studies with human participants or animals performed by any of the authors.

Peer Review. Please note, contrary to the journal's standard single-blind peer review process, as a commentary this article underwent review by a member of the journal's Editorial Board.

Data Availability. Data sharing is not applicable to this article as no datasets were generated or analysed during the current study.

Open Access. This article is licensed under a Creative Commons Attribution-NonCommercial 4.0 International License, which permits any non-commercial use, sharing, adaptation, distribution and reproduction in any medium or format, as long as you give appropriate credit to the original author(s) and the source, provide a link to the Creative Commons licence, and indicate if changes were made. The images or other third party material in this article are included in the article's Creative Commons licence, unless indicated otherwise in a credit line to the material. If material is not included in the article's Creative Commons licence and your intended use is not permitted by statutory regulation or exceeds the permitted use, you will need to obtain permission directly from the copyright holder. To view a copy of this licence, visit http://creativecommons.org/licenses/bync/4.0/. 


\section{REFERENCES}

1. Ahmed SH, et al. Ramadan and diabetes: a narrative reviewand practice update. Diabetes Ther. 2020. https://doi.org/10.1007/s13300-020-00886-y

2. International Diabetes Federation. Diabetes and Ramadan: practical guidance 2016. Available from: https://www.worlddiabetesfoundation.org/sites/de fault/files/IDF\%26DARGuidelinesApril-16-low_0. pdf. Cited 12 May 2020.

3. Beshyah SA. Fasting during the month of Ramadan for people with diabetes: medicine and Fiqh united at last. Ibnosina J Med Biomed Sci. 2009;1(2):58-60.

4. Ghouri N, et al. COVID-19, diabetes and Ramadan. Diabetes Ther. 2020. https://doi.org/10.1007/ s13300-020-00887-x

5. Al-Khawari M, Al-Ruwayeh A, Al-Doub K, Allgrove $\mathrm{J}$. Adolescents on basal-bolus insulin can fast during Ramadan. Pediatr Diabetes. 2010;11(2):96-100.

6. Benbarka MM, Khalil AB, Beshyah SA, Marjei S, Awad SA. Insulin pump therapy in Moslem patients with type 1 diabetes during Ramadan fasting: an observational report. Diabetes Technol Ther. 2010;12(4):287-90.

7. Alamoudi R, Alsubaiee M, Alqarni A, Saleh Y, Aljaser S, Salam A, et al. Comparison of insulin pump therapy and multiple daily injections insulin regimen in patients with type 1 diabetes during Ramadan fasting. Diabetes Technol Ther. 2017;19(6):349-54.

8. Loh HH, Lim LL, Loh HS, Yee A. Safety of Ramadan fasting in young patients with type 1 diabetes: a systematic review and meta-analysis. J Diabetes Investig. 2019;10(6):1490-501.

9. Khalil AB, Beshyah SA, Abu Awad SM, Benbarka MM, Haddad M, Al-Hassan D, et al. Ramadan fasting in diabetes patients on insulin pump therapy augmented by continuous glucose monitoring: an observational real-life study. Diabetes Technol Ther. 2012;14(9):813-8.

10. Kaplan W, Afandi B. Blood glucose fluctuation during Ramadan fasting in adolescents with type 1 diabetes: findings of continuous glucose monitoring. Diabetes Care. 2015;38:e162-e163163.

11. Al-Ozairi E, El Samad A, Al Kandari J, Aldibbiat AM. Intermittent fasting could be safely achieved in people with type 1 diabetes undergoing structured education and advanced glucose monitoring. Front Endocrinol (Lausanne). 2019;10:1-9.

12. Al-Agha AE, Kafi SE, Zain Aldeen AM, Khadwardi $\mathrm{RH}$. Flash glucose monitoring system may benefit children and adolescents with type 1 diabetes during fasting at Ramadan. Saudi Med J. 2017;38(4): 366-71.

13. Elbarbary N. Effectiveness of the low-glucose suspend feature of insulin pump during fasting during Ramadan in type 1 diabetes mellitus. Diabetes Metab Res Rev. 2016;32:623-33.

14. Bally L, Thabit H, Hovorka R. Glucose-responsive insulin delivery for type 1 diabetes: the artificial pancreas story. Int J Pharm. 2018;544(2):309-18.

15. Petrovski G, Al-Khalaf F, Campbell J, Hussain K, Fisher H, Umer F. Glucose control during Ramadan fasting in a teenager with type 1 diabetes on MiniMed 670G hybrid closed-loop system. Acta Diabetol. 2020;57(1):105-7. https://doi.org/10.1007/ s00592-019-01414-6.

16. Lewis D, Leibrand S. Real-world use of open source artificial pancreas systems. J Diabetes Sci Technol. 2016;10:1411.

17. Lewis DM, Swain RS, Donner TW. Improvements in A1C and time-in-range in DIY closed-loop (OpenAPS) users. Diabetes. 2018;67(Suppl 1):352-OR. Available from: https://diabetes.diabetesjournals. org/content/67/Supplement_1/352-OR.abstract. Accessed 20 July 2020.

18. Litchman ML, Lewis D, Kelly LA, Gee PM. Twitter analysis of \#OpenAPS DIY artificial pancreas technology use suggests improved A1C and quality of life. J Diabetes Sci Technol. 2019;13(2):164-70.

19. Street T. \#WeAreNotWaiting at the Type1AndTech Conference. Available from: https://circles-of-blue. winchcombe.org/index.php/2018/11/11/wearenot waiting-at-the-type1andtech-conference/. Accessed 20 July 2020.

20. Wilmot EG, Danne T. DIY artificial pancreas systems: the clinician perspective. Lancet Diabetes Endocrinol. 2020;8(3):183-5.

21. Ahmed SH, Ewins DL, Bridges J, Timmis A, Payne N, Mooney C, et al. Do-It-Yourself (DIY) Artificial Pancreas Systems for Type 1 Diabetes: Perspectives of Two Adult Users, Parent of a User and Healthcare Professionals. Adv Ther. 2020. https://doi.org/10. 1007/s12325-020-01431-w. 